\title{
Integrated Timing of Stroking, Breathing, and Kicking in Front-Crawl Swimming: A Novel Stroke-by-Stroke Approach Using Wearable Inertial Sensors
}

\author{
Silvia Fantozzi ${ }^{1,2, *(\mathbb{D})}$, Vittorio Coloretti ${ }^{1}$, Maria Francesca Piacentini ${ }^{3}\left(\mathbb{D}\right.$, Claudio Quagliarotti $^{3}(\mathbb{D}$, \\ Sandro Bartolomei ${ }^{4}$ (D), Giorgio Gatta ${ }^{5}$ (D) and Matteo Cortesi ${ }^{5, *}$ (D)
}

1 Department of Electrical, Electronic, and Information Engineering "Guglielmo Marconi", University of Bologna, Viale Risorgimento 2, 40136 Bologna, Italy; vittorio.coloretti@studio.unibo.it

2 Health Sciences and Technologies-Interdepartmental Centre for Industrial Research, University of Bologna, Viale Risorgimento 2, 40136 Bologna, Italy

3 Department of Movement, Human and Health Sciences, University of Rome Foro Italico, 00135 Rome, Italy; mariafrancesca.piacentini@uniroma4.it (M.F.P.); c.quagliarotti@studenti.uniroma4.it (C.Q.)

4 Department of Biomedical and Neuromotor Sciences, University of Bologna, 40126 Bologna, Italy; sandro.bartolomei@unibo.it

5 Department for Life Quality Studies, University of Bologna, 40126 Bologna, Italy; giorgio.gatta@unibo.it

* Correspondence: silvia.fantozzi@unibo.it (S.F.); m.cortesi@unibo.it (M.C.); Tel.: +39-051-20988776 (S.F.); +39-051-2088777 (M.C.)

check for

updates

Citation: Fantozzi, S.; Coloretti, V.; Piacentini, M.F.; Quagliarotti, C.;

Bartolomei, S.; Gatta, G.; Cortesi, M. Integrated Timing of Stroking,

Breathing, and Kicking in

Front-Crawl Swimming: A Novel

Stroke-by-Stroke Approach Using

Wearable Inertial Sensors. Sensors

2022, 22, 1419. https://doi.org/

$10.3390 / \mathrm{s} 22041419$

Academic Editor: Jaap van Dieën

Received: 14 January 2022

Accepted: 8 February 2022

Published: 12 February 2022

Publisher's Note: MDPI stays neutral with regard to jurisdictional claims in published maps and institutional affiliations.

Copyright: (C) 2022 by the authors. Licensee MDPI, Basel, Switzerland. This article is an open access article distributed under the terms and conditions of the Creative Commons Attribution (CC BY) license (https:// creativecommons.org/licenses/by/ $4.0 /)$.

\begin{abstract}
Quantitative evaluation of synergic action among the different body segments is fundamental to swimming performance. The aim of the present study was to develop an easy-to-use tool for stroke-by-stroke evaluation of a swimmer's integrated timing of stroking, kicking, and breathing. Twelve swimmers were evaluated during one trial of $100 \mathrm{~m}$ front-crawl swimming at self-selected speed. Five three-axial inertial sensors were mounted on the head, wrists, and ankles. Algorithms for the wrist entry into the water, the lower limb beat during the downward action, and the exit/entry of the face from/into the water were developed. Temporal events identified by video-based technique, using one sagittal moving camera, were assumed as the gold standard. The performance was evaluated in terms of the root-mean-square error, 90th percentile of absolute error, coefficient of variation, Bland-Altman plots, and correlation analysis. Results of all temporal events showed high agreement with the gold standard, confirmed by a root-mean-square error of less than $0.05 \mathrm{~s}$ for absolute temporal parameters and less than $0.7 \%$ for the percentages of the stroke cycle duration, and with correlation coefficients higher than 0.856 . The protocol proposed was not only accurate and reliable, but also user-friendly and as unobtrusive as possible for the swimmer, allowing a stroke-by-stroke analysis during the training session.
\end{abstract}

Keywords: kinematics; wearable device; inertial measurement unit; gyroscope; validation; performance analysis; training monitoring

\section{Introduction}

Swimming performance is strictly related to the synergic action among the different body segments. The analysis of a singular body segment does not completely characterize the actions, and the movement of the limbs cannot be considered independently [1,2]. Depending on swimming speed and athletic skill, swimmers change their coordination between limbs and breathing action [3]. Furthermore, the variability characterization of the synergic action is a way to understand the performance profile [4]. Skilled swimmers seem able to maintain a stable pattern of the stroke parameters to standardize their motor pattern and postpone a technique degradation, and conversely in less experienced swimmers [5].

Quantitative evaluation of synergic action and variability is fundamental to provide reliable information to coaches and athletes. Since 1970, video cameras have been exploited 
for swimmer kinematics analysis from different points of view [6-9]: timing and distance evaluation, velocity of center of mass, 2D/3D coordinates of anatomical landmarks, and 2D/3D joint angular kinematics. However, the use of multicamera systems requires long processing times; therefore, data are not immediately available. Moreover, the expensive and cumbersome camera setup with a restricted acquisition volume made this technology not well-suited for real-time training context. Since 2000, thanks to the development of wearable inertial sensors, these limits were overcome and ecological evaluation was made available by recording stroke-by-stroke data over a long period [10].

Considering front-crawl, the main contribution for force exertion in the water is attributed to the upper limbs [11,12]. The most commonly estimated quantities for the upper limbs using inertial measurement units (IMUs) were stroke count and stroke rate, achieved by locating the sensors on the wrist or/and back and applying a processing algorithm on raw data, with an accuracy between $65 \%$ and $99 \%$ [13-15]. The temporal segmentation of the different phases (entry and catch, pull, push, recovery) within the stroke was achieved: (i) using specific features directly on the raw data of the wrist sensors [16]; (ii) from three sensors positioned on both forearms and lower back [17]; or (iii) using the arm kinematic chain for estimating the joint angular kinematics from the orientation of five sensors on the trunk, arms, and forearms $[18,19]$. Despite its key role within the stroke, the entry event is the most critical instant to be detected by IMU, probably due to high variation related to the swimmer specialization $[20,21]$. From temporal-phase parameters, together with position and velocity of body segments, previous studies investigated start/end propulsion of one arm with respect to the other (coordination index) [17], the interlimb coordination (continuous relative phase) [22], and synchronization [23]. The adaptive role of movement and coordination variability were assessed by using these motor control indexes, highlighting how behavior functionally responds to environmental and task constraints [4,24].

The action of the lower limbs is essential not only for trunk balance and buoyancy [11], but also influences the arms' propulsive action by modifying the hand trajectory and by increasing stroke length [25]. Regarding flutter kicking, the main estimated parameters using IMU were kick count and kick rate, showing reliable results (standard error of the estimate: $5.9 \pm 0.5 \%$ ) [26] and allowing an evaluation of the fatigue effect on this parameter [27]. In both articles, specific features drawn directly from the raw data of the ankle sensor were exploited [26]. However, the synergic action of the lower limbs with respect to the upper limbs or its variability were not investigated.

The breathing frequency or laterality was investigated as an independent variable to estimate the effect on stroke parameters [28]. However, no specific analysis regarding breathing technique was performed using IMUs. The sensor location on the head had several advantages, as it did not affect drag, and measured overall body motion. For this reason, this location was used to estimate the stroke parameters or intracyclic velocity of the swimmer $[29,30]$. Nevertheless, Shell et al. found the accuracy of stroke parameter estimation was not sufficient enough for the training monitoring tool [29].

As previously highlighted, the accuracy of temporal parameters for upper and lower limb actions were investigated independently. To give a complete characterization of the swimmer's action and to understand the coordination between the movements of the head and the upper and lower limbs, the combination of these features should be taken into account. Nevertheless, a combined timing analysis of stroking, kicking, and breathing actions has not been evaluated. Furthermore, this type of analysis must be performed in ecological conditions, and the protocol should be friendly and plain enough to be used by coaches during training sessions. For this reason, the aim of the present study was to develop and validate an easy-to-use tool for stroke-by-stroke evaluation of a swimmer's integrated timing of stroking, kicking, and breathing. The validation of temporal parameter estimation for the upper limbs, the lower limbs, and the breathing action were performed singularly. We hypothesized that IMU technologies would allow the estimation of swimmer timings regarding stroking, kicking, and breathing with sufficient accuracy for the purpose. 


\section{Materials and Methods}

\subsection{Protocol: Participants, Trials and Instrumentation}

Twelve male swimmers (age: $19.1 \pm 2.3$ years; mass: $76.7 \pm 3.7 \mathrm{~kg}$; height: $179.0 \pm 5.2 \mathrm{~cm}$; level: $686 \pm 82.6$ FINA points long course, Tier 3 [31]) were evaluated during front-crawl swimming at self-selected speed in a $25 \mathrm{~m}$ swimming pool. For each participant, one trial of $100 \mathrm{~m}$ was acquired.

Five triaxial IMUs (Cometa, Milano, Italy) equipped with an accelerometer (sensitivity: $1563 \mathrm{mV} / \mathrm{g}$; full scale: $\pm 16 \mathrm{~g}$ ) and gyroscope (sensitivity: $1.3 \mathrm{mV} / \mathrm{g}$; full scale: $\pm 2000^{\circ} / \mathrm{s}$ ) were calibrated at the beginning of each acquisition session. Successively, IMUs were attached to the head (in the occipital zone between the supreme and superior nuchal lines), forearms (about two centimeters above the styloid), and shanks (about two centimeters above lateral malleolus). Then, 3D acceleration and 3D angular velocity were acquired from each sensor with a sampling frequency of $285 \mathrm{~Hz}$. Biadhesive tape $(0.05 \mathrm{~m} \times 25 \mathrm{~m}$ Eurocel, SICAD S.p.A. ITALIA, Varese, Italy) was used for all IMUs. In addition, for the head, the sensor was inserted between two swim caps, and for the limbs, a coband (BSN Medical Co-Plus ${ }^{\circledR}$ Lf $0.075 \mathrm{~m} \times 4.5 \mathrm{~m}$, BSN medical GmbH, Hamburg, Germany) was used to firmly fix the sensors. The IMU sensor for the head had the $X$-axis aligned with the longitudinal axis of the skull pointing upward, the $Y$-axis with the transverse axis pointing to the left, and the Z-axis consequently aligned. The IMU sensor for the wrist had the X-axis aligned with the longitudinal axis of the forearm pointing proximally, the $Y$-axis with the radioulnar axis, and the $Z$-axis consequently aligned. The IMU sensor for the ankle was aligned with the $X$-axis aligned to the sagittal axis of the tibia pointing forward, the $Y$-axis to the longitudinal axis pointing proximally, and the Z-axis consequently aligned. Details of the IMU attachment and axes alignment are reported on the right in Figure 1.

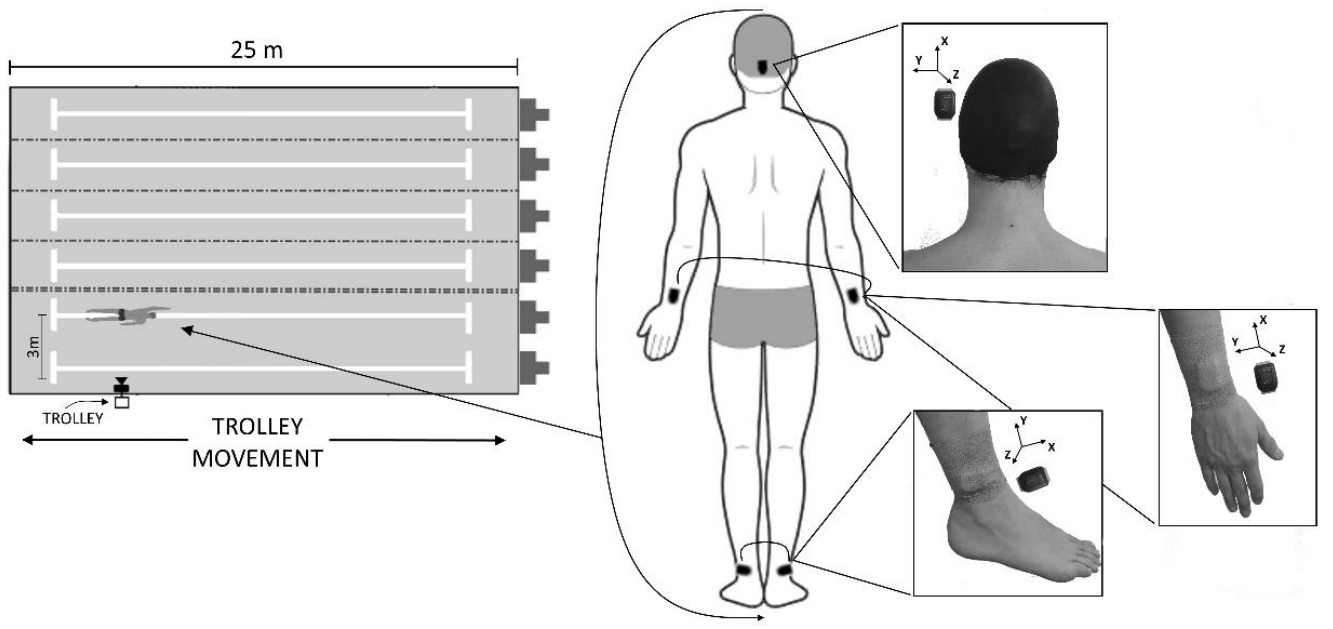

Figure 1. Single sagittal camera on trolley followed the athlete at similar velocity during $100 \mathrm{~m}$ front-crawl swimming (left). Positioning of wearable inertial sensors on head, wrist, and ankle (right). Alignment of axes $(X, Y$, and $Z)$ of reference system are shown for each position.

The swimming trials were also filmed using a single moving sagittal video camera (Hero7, GoPro, San Mateo, CA, USA, sf $=240 \mathrm{~Hz}, 1920 \times 1080$ pixel resolution) for the event detection gold standard (TLC). The camera was held on a sagittal plane by an operator following the swimmer at similar velocity using a trolley (Figure 1 on the left). Stroking, breathing, and kicking temporal events were clearly visible on the images of the video for all the strokes within the lap. The same expert operator identified all the events in the video. The sensors' flashing LEDs were video-recorded to synchronize data acquired using IMUs and video recording. 


\subsection{Data Analysis}

\subsubsection{Stroking}

In the present analysis, the entry of the wrist into the water (WRIST ENTRY $_{\text {) was used for }}$

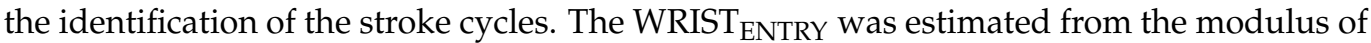
the angular jerk measured by the wrist sensor. The modulus was preferred with respect to a specific axis in order to be as independent as possible from the specific swimmer's technique. First, a clearly identifiable instant of the recovery phase was detected by calculating the maximum value of the angular velocity about the radioulnar direction ( $Y$-axis). Then, the modulus of the angular jerk was calculated by performing the double differentiation of the

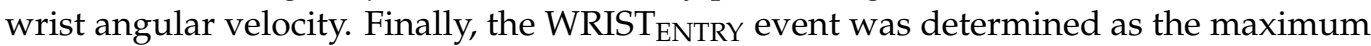
of the jerk modulus between two previously identified successive recovery phases. An example pattern of the quantities used for the algorithm and the estimated WRIST ENTRY $_{\text {. }}$ event are given in Figure $2 a, b$, respectively.

a) STROKING

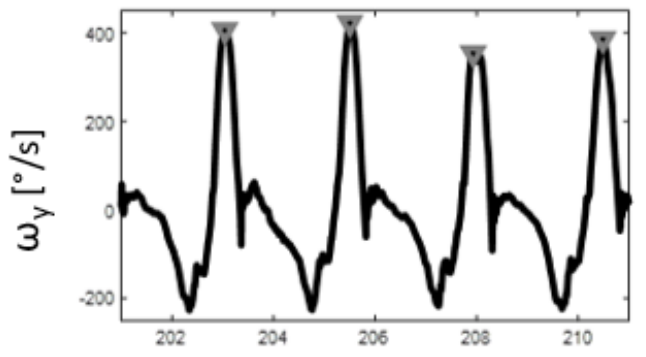

b) STROKING

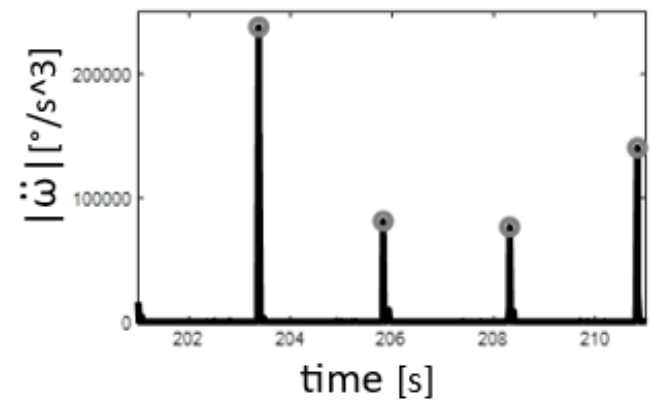

c) KICKING

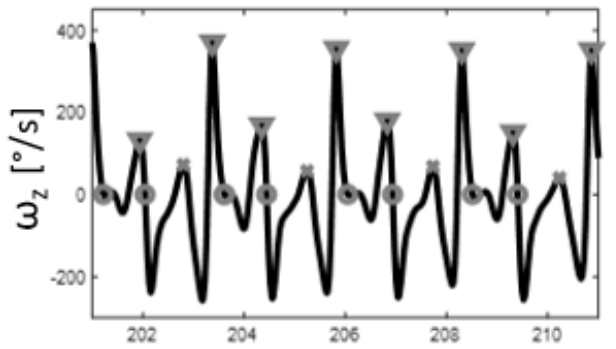

d) BREATHING

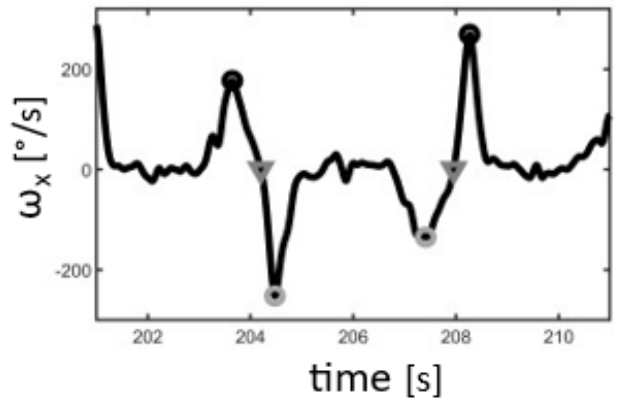

Figure 2. A typical pattern of four strokes for one swimmer. Stroking events using the wrist IMU: (a) angular velocity of $Y$ axis with maximum for recovery phase identification (grey trian-

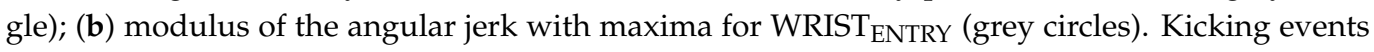
using the ankle IMU: (c) angular velocity of Z-axis with propulsive (grey triangle) and buoyancy

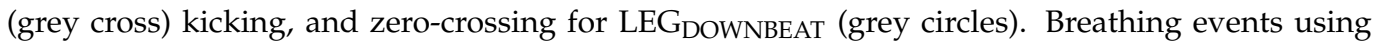
the head IMU: (d) angular velocity of $X$-axis with zero-crossing (grey triangle) between maximum/minimum (black/grey circle) for HEAD EXIT $_{\text {and HEAD }}$ ENTRY (before and after zero-crossing maximum/minimum events, respectively).

\subsubsection{Kicking}

For the time characterization of the leg action, the event corresponding to the beat

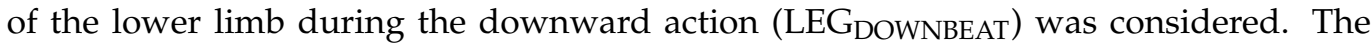
mediolateral angular velocity of the IMUs on the ankle was used (Z-axis). To estimate the beat, the zero-crossing of the angular velocity was employed, and to distinguish the downfrom the up-beat, the orientation of the axis and the sign before and after the zero-crossing were taken into account. An example pattern of the quantities used for the algorithm and the estimated LEG DOWNBEAT event is given in Figure 2c.

In the analysis, two types of kicking actions were observed: propulsive and buoyancy kicks. They were first visually inspected considering the regularity of the leg movement: in some swimmers, the pattern of the angular velocity had similar maximum values through- 
out the trials, while others showed two/three maximum comparable values followed by one at a clearly distinguished lower maximum value. This last beat was interpreted as a beat performed for buoyancy purposes and not for propulsion. For this reason, this maximum was not included in the analysis: an adaptable threshold was calculated from the mean plus two standard deviations of all maxima below $100 \mathrm{deg} / \mathrm{s}$ of each athlete. The starting value of $100 \mathrm{deg} / \mathrm{s}$ and the results of the exclusion were established from the video analysis, in which the difference between propulsive and buoyancy kicking was clearly distinguished.

\subsubsection{Breathing}

Regarding the breathing analysis, the exit and the entry of the face from/into the water were considered (HEAD EXIT $_{\text {and HEAD }}$ ENTRY, respectively). The longitudinal angular velocity of the IMUs on the head was used (X-axis). From the zero-crossing of this variable, the start of the face rotation out of the water was first identified. Successively, the maximum/minimum values before and after the zero-crossing corresponded to the exit/entry of the head depending on the breathing side: the event before the zero-crossing was the exit, the event after was the entry. An example pattern of the quantities used for the algorithm and of the estimated HEAD ${ }_{\text {EXIT }}$ and HEAD ${ }_{\text {ENTRY }}$ events is given in Figure $2 \mathrm{~d}$.

\subsubsection{Timing}

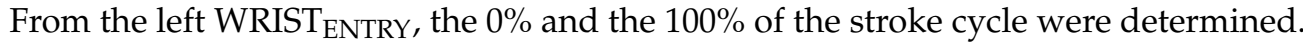
WRIST $_{\text {ENTRY }}$ contralateral arm, LEG $_{\text {DOWNBEAT, }}$ HEAD EXIT, and HEAD ENTRY events were expressed in percentage of the stroke cycle.

Data processing of the three above algorithms and timing analysis were performed in MATLAB (MathWorks, Natick, MA, USA, 2019).

\subsubsection{Statistical Analysis}

All data were expressed as means $( \pm S D)$. The Shapiro-Wilk test was used to confirm

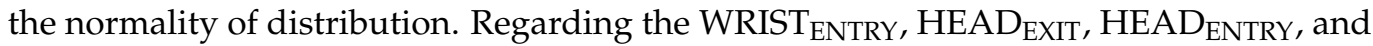
LEG $_{\text {DOWNBEAT }}$ absolute temporal values and percentages of the stroke cycle, the accuracy of the IMU algorithms in comparison to TLC were determined by the root-mean-square error (RMSE [32]) and 90th percentile of absolute error, and the reliability was computed using the coefficient of variation (CV) and typical error of measurement (TEM). Considering the sampling frequency of the video acquisition, a minimum detectable difference threshold of $0.005 \mathrm{~s}$ was considered. To complement the agreement analyses between the two techniques, Bland-Altman plots and a correlation analysis were created. To calculate the concurrent validity, the bivariate Pearson product moment correlation coefficient was used. The correlation magnitude was interpreted as 0.1 (low), 0.3 (moderate), 0.5 (large), 0.7 (very high), and 0.9 (nearly perfect) as proposed by Hopkins et al. [33]. All statistical tests were performed using the software SPSS version 20.0 (SPSS, Chicago, IL, USA) and Microsoft Excel 2010. The level of statistical significance was set at $p<0.05$.

\section{Results}

Regarding absolute temporal variables, the average RMSEs and 90th percentile of absolute errors for IMU vs. TLC were $<0.005 \mathrm{~s}$ and $0.041 \mathrm{~s}$ for WRIST ENTRY, $0.005 \mathrm{~s}$ and $0.197 \mathrm{~s}$ for HEAD EXIT, $<0.005 \mathrm{~s}$ and $0.060 \mathrm{~s}$ for HEADENTRY, and $<0.005 \mathrm{~s}$ and $0.040 \mathrm{~s}$ for LEG $_{\text {DOWNBEAT. The CVs for IMU and TLC were } 0.068 \text { and } 0.069 \text { for WRIST }}$ ENTRY,

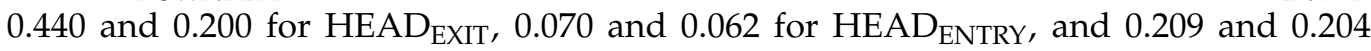
for $L_{E G}$ DOWnBEAT. The respective TEMs for IMU and TLC were 0.083 and $0.083 \mathrm{~s}$ for WRIST $_{\text {ENTRY, }} 0.147$ and $0.085 \mathrm{~s}$ for HEAD $\mathrm{EXIT}, 0.081$ and $0.073 \mathrm{~s}$ for HEAD $\mathrm{ENTRY}_{\text {, and } 0.240}$ and $0.232 \mathrm{~s}$ for LEG DOWNBEAT.

Bland-Altman and correlation procedures were employed to determine measurement bias (Figure 3). The limits of agreement (LoA) between the IMU and TLC had a range of -0.054 to $0.053 \mathrm{~s}$ for WRIST $\mathrm{ENTRY},-0.072$ to $0.265 \mathrm{~s}$ for HEAD $\mathrm{EXIT},-0.054$ to $0.074 \mathrm{~s}$ 
to for HEAD ENTRY, and $-0.049 \mathrm{~s}$ to $0.050 \mathrm{~s}$ for $\mathrm{LEG}_{\mathrm{DOWNBEAT}}$ with nonsignificant biases of $<0.005 \mathrm{~s}, 0.097 \mathrm{~s}, 0.010 \mathrm{~s}$ and $<0.005 \mathrm{~s}$, respectively. In addition, the Pearson product moment correlation coefficient showed nearly perfect correlation between the IMU and

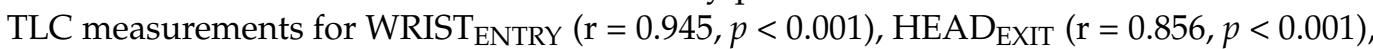
$\operatorname{HEAD}_{\text {ENTRY }}(\mathrm{r}=0.916, p<0.001)$, and LEG DOWNBEAT $(\mathrm{r}=0.998, p<0.001)$. In general, IMU algorithms showed consistent results for all analyzed temporal events.
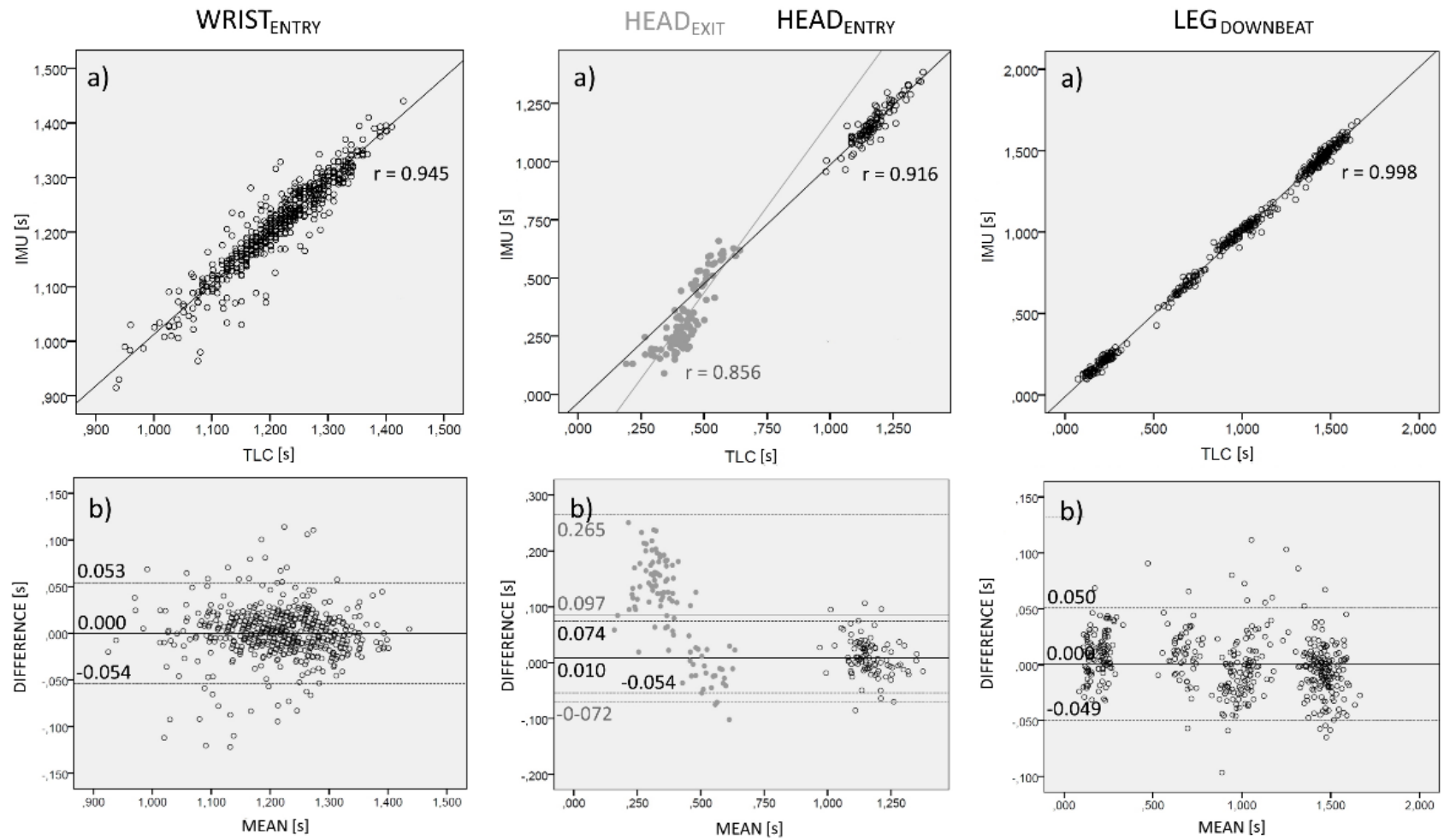

Figure 3. Linear regression analysis (a) and Bland-Altman plots (b) for WRIST ENTRY (open circles), HEAD EXIT (grey filled circle), HEAD ENTRY (open circle), and LEG comparing measurements with IMUs and video-based technique (TLC). In (b), central lines represent the intermethod differences (biases). Upper and lower dotted lines represent the $95 \%$ limits of agreement (bias $\pm 1.96 \mathrm{SD}$ of the differences).

The average timing of the analyzed temporal events in percentage of the stroke duration are reported in Table 1. Regarding timing, the respective average RMSEs and 90th percentile of absolute errors for IMU vs. TLC were 0.1 and $1.5 \%$ for WRIST ENTRY, 0.7 and $8.0 \%$ for HEAD $\mathrm{EXIT}_{1}, 0.1$ and $2.2 \%$ for HEAD ENTRY, and 0.1 and $1.8 \%$ for LEGDOWNBEAT. The respective CVs for IMU and TLC were 0.0 and 0.0 for WRIST ENTRY, 0.4 and 0.1 for HEAD EXIT, $_{0.0}$ and 0.0 for HEAD ENTRY, and 0.1 and 0.1 for LEG $_{\text {DOWNBEAT. The respec- }}$ tive TEMs for IMU and TLC in percentage of the stroke duration were 2.2 and 2.1 for

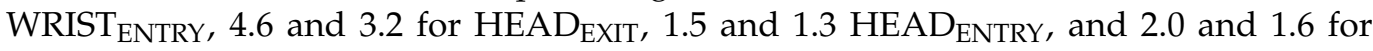

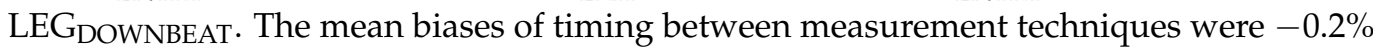

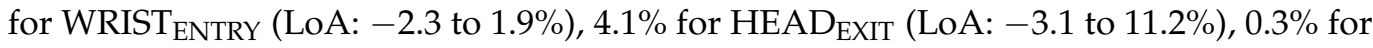

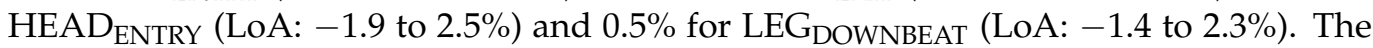
timings of the analyzed temporal events in percentage of the stroke duration using IMUs and TLC for a single participant are reported in Figure 4. 
Table 1. Mean \pm SD timing of the analyzed temporal events in percentage of the stroke duration when comparing measurements with IMUs and video-based technique (TLC). The stroke duration was normalized to left WRIST ENTRY $_{\text {events. }}$

\begin{tabular}{|c|c|c|}
\hline Temporal Events & IMUs & TLC \\
\hline First right $\mathrm{LEG}_{\text {DOWNBEAT }}(\%)$ & $2.3 \pm 7.6$ & $3.1 \pm 6.9$ \\
\hline First left LEG DOWNBEAT (\%) & $15.5 \pm 9.4$ & $16.0 \pm 9.3$ \\
\hline Right HEAD EXIT $(\%)$ & $15.9 \pm 5.7$ & $18.7 \pm 2.8$ \\
\hline Right HEAD ENTRY $(\%)$ & $48.0 \pm 2.0$ & $48.4 \pm 2.0$ \\
\hline Right WRIST ENTRY $(\%)$ & $49.3 \pm 2.2$ & $49.0 \pm 2.1$ \\
\hline Second left LEG DOWNBEAT (\%) & $53.7 \pm 8.3$ & $53.0 \pm 7.6$ \\
\hline Left HEAD $D_{\text {EXIT }}(\%)$ & $58.2 \pm 3.5$ & $65.2 \pm 3.5$ \\
\hline Second right LEG DOWNBEAT $(\%)$ & $64.1 \pm 8.2$ & $64.8 \pm 8.7$ \\
\hline Left HEAD ENTRY $(\%)$ & $96.6 \pm 1.0$ & $96.8 \pm 0.6$ \\
\hline
\end{tabular}

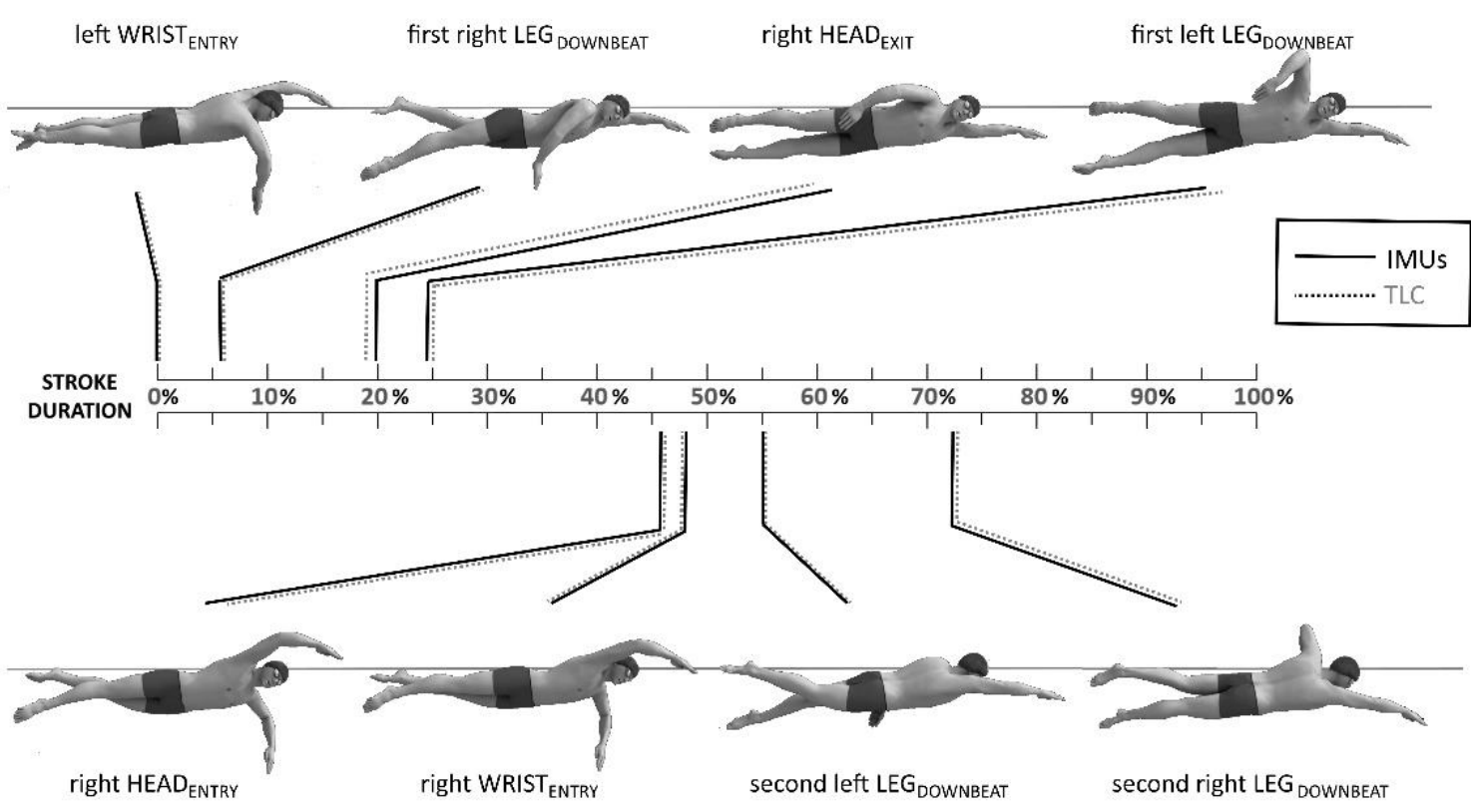

Figure 4. The timing of the analyzed temporal events in percentage of the stroke duration using IMUs (solid black lines) and video-based technique (TLC, dotted grey lines) for a single participant.

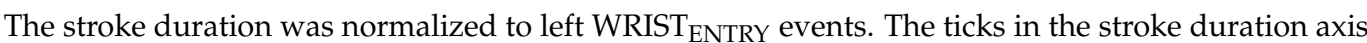
correspond to $5 \%$

\section{Discussion}

A complete characterization of a swimmer's action requires not only an independent analysis of temporal parameters for the head and upper/lower limb actions, but also an integration of these features. Furthermore, the assessment must be performed for the whole motor task and not only for a few strokes. The present study developed an integrated stroke-by-stroke tool for the assessment of the stroking, kicking, and breathing timing in front-crawl swimming using wearable inertial sensors. To test our hypothesis, data for 12 athletes were collected using IMUs attached to the head, wrists, and ankles, and timing features were compared with video analysis. Results of all temporal events showed high agreement with the gold standard video-based technique, confirmed by an RMSE less than $0.05 \mathrm{~s}$ for absolute temporal parameters and less than $0.7 \%$ for the percentage of the stroke duration.

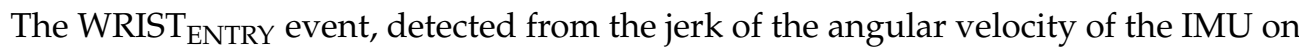
the wrist, showed high agreement with TLC both in terms of absolute temporal value (bias: $<0.005 \mathrm{~s}$, LoA: $-0.0054 \mathrm{~s}$ to $0.053 \mathrm{~s}$ ) and in terms of percentage of stroke cycle duration (bias: $-0.2 \%$, LoA: $-2.3 \%$ to $1.9 \%)$ with nearly perfect correlation $(r=0.945)$. The present study's results were comparable with, and for some parameters, even more accurate than 
those of previous studies. In more detail, when arm kinematic chain and multiple IMUs were exploited, a bias lower than $1.4 \%$, a 95\% LoA lower than 7.7\%, an RMSE lower than $3.5 \%$, and an $\mathrm{r}$ higher than 0.81 were found for entry, catch, pull, push, and recovery phases [19]. When three IMUs and raw data sensors were used, the 95\% LoA for stroke phase durations was always lower than $7.9 \%$ of the stroke cycle duration [17]. Furthermore, differently from previous studies, the proposed method did not require any specific body segment alignment calibration or any anthropometric measurement, such as the body segment length, and involved the use of only one sensor per limb. In this context, previous studies highlighted how the hand entry was highly dependent on the swimmer's technique: palmar-dorsal acceleration can exhibit a high or near-zero value depending on a flatter or sharper pitch angle of the hand entry [16]. For this reason, in the developed algorithm, the modulus of the jerk was used while taking into account all three components of the physical quantity. From the data acquired, every single stroke was automatically detected by the present algorithm without any operator supervision.

The algorithm for the analysis of the kicking timing of the lower limbs exhibited high agreement (bias: $<0.005 \mathrm{~s}$, LoA: $-0.049 \mathrm{~s}$ to $0.050 \mathrm{~s}$; bias: $0.5 \%$, LoA: $-1.4 \mathrm{~s}$ to $2.3 \mathrm{~s}$ ) and nearly perfect correlation $(r=0.998)$ with the video-based technique. The algorithm proposed by Fulton et al. for counting the kicks [26] was slightly modified for the estimation of timing in absolute temporal value and in percentage of the stroke cycle. The modification was necessary, as two types of kicking action were noticed in our study participants. Together with the propulsive kicks, clearly distinguishable buoyancy kicks were observed in the videos. In this latter case, the lower limb of the swimmer exhibited a small beat, mainly due to the rotation of the trunk/pelvis segment and not to a strong action of the leg. From observing the angular velocity of the IMU on the ankle, the buoyancy kicks clearly showed lower peak values, and the modification was developed to exclude them from the analysis. These issues were not reported by Fulton et al., most likely because, differently from the present study, maximal-effort trials were acquired [26,27]. No comparison with existing literature regarding timing features could be performed, as the only previous study that exploited IMUs for kicking action analysis investigated the performance of the algorithms only for counts and rate.

The breathing features investigated showed different levels of accuracy depending

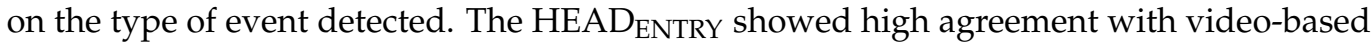
techniques both in terms of absolute temporal quantity (bias: $0.01 \mathrm{~s}$; LoA: $-0.054 \mathrm{~s}$ to $0.074 \mathrm{~s}$ ) and in terms of percentage of the stroke duration (bias: $0.5 \%$; LoA: $-1.9 \mathrm{~s}$ to $2.5 \mathrm{~s}$ ), with nearly high correlation coefficient $(r=0.916, p<0.001)$. In addition, the HEAD EXIT event detection exhibited a good degree of agreement. In more detail, the larger bias found $(0.097 \mathrm{~s} ; 4.1 \%)$ with respect to HEAD ENTRY could be explained by a fixed temporal shift between the feature extracted from the longitudinal angular velocity and the exit of the face from the water identified in the video. Furthermore, the LoAs of HEAD EXIT showed higher values $(-0.072 \mathrm{~s}$ to $0.027 \mathrm{~s} ;-3.1 \%$ to $11.2 \%$ ) and slightly less reliability for the analysis of this temporal feature with respect to the entry. However, considering the high

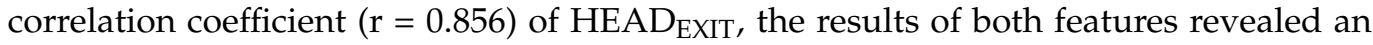
accuracy sufficient for analysis purposes. Indeed, the error value of HEAD $\mathrm{EXIT}_{\mathrm{T}}$ expressed in percentage of the stroke cycle duration was, in any case, lower than previous studies investigating temporal stroke features. Specific comparison with existing literature could not be performed, as the present study was the first to validate an algorithm for timing breathing detection using IMUs. However, it has been demonstrated how investigating the breathing action in front-crawl swimming is fundamental not only to the energetic efficiency, but also to the effect on upper limb kinematics and overall technique [28]. For this reason, a reliable algorithm for the detection of breathing timing allows monitoring of the effects of fatigability or different techniques on the breathing timing, in addition to the intratrial variability of these effects.

Head and upper/lower limb actions of the swimmer during front-crawl swimming were accurately and repeatably identified in terms of absolute temporal value (Figure 3). 
Furthermore, the stroking, kicking, and breathing features were integrated with the stroke cycle duration (Table 1 and Figure 4), allowing a reliable combined timing analysis of the action of all principal factors in front-crawl using the minimum number of inertial sensors. Swimming is a cyclic activity, and the synchronization between the head and upper/lower arms can only be optimal if it can be maintained along consecutive stroke cycles [23]. In order to produce the propelling effects, the stroking, kicking, and breathing must occur at the same relative time in each stroke. The integrated timing analysis proposed in this study could enable swimming coaches to continuously check the whole timing technique of the swimmers during the training session.

The accuracy and repeatability findings must be considered reliable for the motor task and level of swimmer analyzed. Further investigations will explore the generalization of the results for different coordination models of technique, performance level of swimmers, and different swimming techniques. It must also be highlighted another limitation in the stroking was identified by the entry of the wrist into the water, as the IMU was attached to the wrist. Thus, with the aim of limiting the swimmer burden, the catch event more strictly related to propulsion was not possible to be detected. Coaches could take advantage of the proposed tool to continuously check the whole timing technique and monitor the effects of fatigue or different techniques on the coordination timing.

In conclusion, a protocol for integrated analysis of stroking, kicking, and breathing using inertial sensors in front-crawl swimming was developed and validated in comparison with a video-analysis technique. All accuracy parameters investigated (RMSE, bias, LoA, and correlation) highlighted high agreement with the gold standard. Furthermore, the protocol proposed was user-friendly and as unobtrusive as possible for the swimmer, allowing a stroke-by-stroke analysis during the training session.

Author Contributions: Conceptualization, S.F., G.G., M.F.P., S.B. and M.C.; methodology, S.F. and M.C.; software, V.C.; validation, S.F., M.C., V.C., C.Q. and G.G.; data curation, V.C., M.C., C.Q. and S.B.; writing-original draft preparation, S.F. and M.C.; writing-review and editing, V.C., M.F.P., S.B., C.Q. and G.G.; supervision, S.F., S.B., G.G., M.F.P. and M.C. All authors have read and agreed to the published version of the manuscript.

Funding: This research received no external funding.

Institutional Review Board Statement: The study was conducted in accordance with the Declaration of Helsinki, and approved by the Ethics Committee of University of Bologna (protocol code 0196686; date of approval 22 September 2020).

Informed Consent Statement: Informed consent was obtained from all subjects involved in the study.

Data Availability Statement: The data presented in this study are available upon request from the corresponding authors. The data are not publicly available due to privacy restrictions.

Conflicts of Interest: The authors declare no conflict of interest.

\section{References}

1. Zamparo, P.; Cortesi, M.; Gatta, G. The Energy Cost of Swimming and Its Determinants. Eur. J. Appl. Physiol. 2020, 120, 41-66. [CrossRef] [PubMed]

2. Seifert, L.; Carmigniani, R. Coordination and Stroking Parameters in the Four Swimming Techniques: A Narrative Review. Sports Biomech. 2021, 1-17. [CrossRef] [PubMed]

3. Seifert, L.; Chollet, D.; Allard, P. Arm Coordination Symmetry and Breathing Effect in Front Crawl. Hum. Mov. Sci. 2005, 24, 234-256. [CrossRef]

4. Cortesi, M.; Di Michele, R.; Fantozzi, S.; Bartolomei, S.; Gatta, G. Arm-Stroke Descriptor Variability during 200-m Front Crawl Swimming. Sensors 2021, 21, 324. [CrossRef]

5. Seifert, L.; Chollet, D.; Chatard, J.C. Kinematic Changes during a 100-m Front Crawl: Effects of Performance Level and Gender. Med. Sci. Sports Exerc. 2007, 39, 1784-1793. [CrossRef] [PubMed]

6. Mooney, R.; Corley, G.; Godfrey, A.; Osborough, C.; Quinlan, L.R.; ÓLaighin, G. Application of Video-Based Methods for Competitive Swimming Analysis: A Systematic Review. Sport Exerc. Med. Open J. 2015, 1, 133-150. [CrossRef]

7. Magalhaes, F.A.; Sawacha, Z.; Michele, R.D.; Cortesi, M.; Gatta, G.; Fantozzi, S. Effectiveness of an Automatic Tracking Software in Underwater Motion Analysis. J. Sports Sci. Med. 2013, 12, 660-667. 
8. Ceccon, S.; Ceseracciu, E.; Sawacha, Z.; Gatta, G.; Cortesi, M.; Cobelli, C.; Fantozzi, S. Motion Analysis of Front Crawl Swimming Applying CAST Technique by Means of Automatic Tracking. J. Sports Sci. 2013, 31, 276-287. [CrossRef]

9. Ceseracciu, E.; Sawacha, Z.; Fantozzi, S.; Cortesi, M.; Gatta, G.; Corazza, S.; Cobelli, C. Markerless Analysis of Front Crawl Swimming. J. Biomech. 2011, 44, 2236-2242. [CrossRef]

10. Mooney, R.; Corley, G.; Godfrey, A.; Quinlan, L.; ÓLaighin, G. Inertial Sensor Technology for Elite Swimming Performance Analysis: A Systematic Review. Sensors 2015, 16, 18. [CrossRef]

11. Gatta, G.; Cortesi, M.; Di Michele, R. Power Production of the Lower Limbs in Flutter-Kick Swimming. Sports Biomech. 2012, 11, 480-491. [CrossRef]

12. Bilinauskaite, M.; Mantha, V.R.; Rouboa, A.I.; Ziliukas, P.; Silva, A.J. Computational Fluid Dynamics Study of Swimmer's Hand Velocity, Orientation, and Shape: Contributions to Hydrodynamics. BioMed Res. Int. 2013, 2013, 140487. [CrossRef] [PubMed]

13. Bächlin, M.; Tröster, G. Swimming Performance and Technique Evaluation with Wearable Acceleration Sensors. Pervasive Mob. Comput. 2012, 8, 68-81. [CrossRef]

14. Siirtola, P.; Laurinen, P.; Roning, J.; Kinnunen, H. Efficient Accelerometer-Based Swimming Exercise Tracking. In Proceedings of the 2011 IEEE Symposium on Computational Intelligence and Data Mining (CIDM), Paris, France, 11-15 April 2011 ; pp. 156-161.

15. Chakravorti, N.; Le Sage, T.; Slawson, S.E.; Conway, P.P.; West, A.A. Design and Implementation of an Integrated Performance Monitoring Tool for Swimming to Extract Stroke Information at Real Time. IEEE Trans. Human-Mach. Syst. 2013, 43, 199-213. [CrossRef]

16. Ohgi, Y.; Yasumura, M.; Ichikawa, H.; Miyaji, C. Analysis of Stroke Technique Using Acceleration Sensor IC in Freestyle Swimming. Eng. Sport 2000, 503-511.

17. Dadashi, F.; Crettenand, F.; Millet, G.P.; Seifert, L.; Komar, J.; Aminian, K. Automatic Front-Crawl Temporal Phase Detection Using Adaptive Filtering of Inertial Signals. J. Sports Sci. 2013, 31, 1251-1260. [CrossRef] [PubMed]

18. Mangia, A.L.; Cortesi, M.; Fantozzi, S.; Giovanardi, A.; Borra, D.; Gatta, G. The Use of IMMUs in a Water Environment: Instrument Validation and Application of 3D Multi-Body Kinematic Analysis in Medicine and Sport. Sensors 2017, 17, 927. [CrossRef]

19. Cortesi, M.; Giovanardi, A.; Gatta, G.; Mangia, A.L.; Bartolomei, S.; Fantozzi, S. Inertial Sensors in Swimming: Detection of Stroke Phases through 3D Wrist Trajectory. J. Sports Sci. Med. 2019, 18, 438-447. [PubMed]

20. McCabe, C.B.; Psycharakis, S.; Sanders, R. Kinematic Differences between Front Crawl Sprint and Distance Swimmers at Sprint Pace. J. Sports Sci. 2011, 29, 115-123. [CrossRef] [PubMed]

21. Cortesi, M.; Fantozzi, S.; Gatta, G. Effects of Distance Specialization on the Backstroke Swimming Kinematics. J. Sports Sci. Med. 2012, 11, 526-532.

22. Seifert, L.; L'Hermette, M.; Komar, J.; Orth, D.; Mell, F.; Merriaux, P.; Grenet, P.; Caritu, Y.; Hérault, R.; Dovgalecs, V.; et al. Pattern Recognition in Cyclic and Discrete Skills Performance from Inertial Measurement Units. Procedia Eng. 2014, 72, 196-201. [CrossRef]

23. Mezêncio, B.; Pinho, J.P.; Huebner, R.; Vilas-Boas, J.P.; Amadio, A.C.; Serrão, J.C. Overall Indexes of Coordination in Front Crawl Swimming. J. Sports Sci. 2020, 38, 910-917. [CrossRef] [PubMed]

24. Guignard, B.; Rouard, A.; Chollet, D.; Seifert, L. Behavioral Dynamics in Swimming: The Appropriate Use of Inertial Measurement Units. Front. Psychol. 2017, 8, 383. [CrossRef] [PubMed]

25. Gourgoulis, V.; Boli, A.; Aggeloussis, N.; Toubekis, A.; Antoniou, P.; Kasimatis, P.; Vezos, N.; Michalopoulou, M.; Kambas, A.; Mavromatis, G. The Effect of Leg Kick on Sprint Front Crawl Swimming. J. Sports Sci. 2014, 32, 278-289. [CrossRef]

26. Fulton, S.K.; Pyne, D.B.; Burkett, B. Validity and Reliability of Kick Count and Rate in Freestyle Using Inertial Sensor Technology. J. Sports Sci. 2009, 27, 1051-1058. [CrossRef]

27. Fulton, S.K.; Pyne, D.B.; Burkett, B. Quantifying Freestyle Kick-Count and Kick-Rate Patterns in Paralympic Swimming. J. Sports Sci. 2009, 27, 1455-1461. [CrossRef] [PubMed]

28. McCabe, C.B.; Sanders, R.H.; Psycharakis, S.G. Upper Limb Kinematic Differences between Breathing and Non-Breathing Conditions in Front Crawl Sprint Swimming. J. Biomech. 2015, 48, 3995-4001. [CrossRef]

29. Shell, S.J.; Clark, B.; Broatch, J.R.; Slattery, K.; Halson, S.L.; Coutts, A.J. Is a Head-Worn Inertial Sensor a Valid Tool to Monitor Swimming? Int. J. Sports Physiol. Perform. 2021, 16, 1901-1904. [CrossRef]

30. Butterfield, J.; Tallent, J.; Patterson, S.D.; Jeffries, O.; Howe, L.; Waldron, M. The Validity of a Head-Worn Inertial Sensor for Measurements of Swimming Performance. Mov. Sport Sci. 2021, 114, 3-8. [CrossRef]

31. McKay, A.K.A.; Stellingwerff, T.; Smith, E.S.; Martin, D.T.; Mujika, I.; Goosey-Tolfrey, V.L.; Sheppard, J.; Burke, L.M. Defining Training and Performance Caliber: A Participant Classification Framework. Int. J. Sports Physiol. Perform. 2021, 17, 317-331. [CrossRef]

32. Hopkins, W.G. Linear Models and Effect Magnitudes for Research, Clinical and Practical Applications. Sportscience 2010, 14, 49-59.

33. Hopkins, W.G.; Marshall, S.W.; Batterham, A.M.; Hanin, J. Progressive Statistics for Studies in Sports Medicine and Exercise Science. Med. Sci. Sports Exerc. 2009, 41, 3-12. [CrossRef] [PubMed] 